\title{
PROGESTERONA Y PREGNANDIOL
}

\author{
Dr. Fernando Cardona Arango \\ Dr. Hebert Aristizábal Bustamante
}

\section{INTRODUCCION}

Todo progreso científico en el campo endocrinológico que permita una dosificación más simple, rápida y exacta de una hormona, ya sea en su forma activa circulante, ya sea en su metabolito de excreción, trae como consecuencia lógica un mayor conocimiento de la fisiología y de la patología del organismo en relación con esa hormona, y por consiguiente dichas dosificaciones serán otra valiosa ayuda que tendrá el clínico para saber el estado endocrino de su paciente.

En el campo de la obstetricia y la ginecología, los recientes avances en la dosificación del pregnandiol han permitido que su fiel valoración se ponga más al alcance del clínico y que realmente puedan emitirse nuevos conceptos en relación con la progesterona, pues los amplios límites de ayer de las cifras normales en el ciclo menstrual y el embarazo se han reducido hoy a un mínimo con la eliminación de los cromógenos por medios cromatográficos, y así las cifras actuales de la pregnandioluria expresan conceptos tan definidos y precisos que hacen posibles y aún necesarios, la elaboración de más estudios sobre la progesterona pura en esta época de los progestágenos sin- téticos orales de gran acción progestacional.

Nos referiremos en el presente trabajo primero a algunas consideraciones químicas y biológicas sobre el metabolismo de la progesterona, luego comentaremos una nueva técnica cromatográfica que logra la dosificación del pregnandiol en la orina, y finalmente nos ocuparemos de las cifras normales del pregnandiol en el ciclo menstrual, en el embarazo y en la amenaza de aborto.

\section{CONSIDERACIONES QUIMICAS $Y$ BIOLOGICAS SOBRE EL METABOLIS- MO DE LA PROGESTERONA}

a) Metabolismo: La progesterona segregada por el ovario y la suprarenal, así como la placentaria y la de origen exógeno es inactivada a nivel del hígado en donde se efectúa su reducción y conjugación y se elimina como pregnandiol por la orina $y$ las materias fecales.

El primer paso en su metabolismo (23) se efectúa en el grupo delta 4 , 3 ceto; tomando un hidrógeno $(\mathrm{H})$ en el carbón 5, y suprimiendo el doble enlace del carbón 4, y así la - 4 Pregnen-3,20 Diona - progesterona se transforma en - Pregnan-3,20 Diona y Alopregnan 3, 20 Diona - de- 
pendiendo ello de la posición "cis" (Beta), ó "trans" (alfa) del hidrógeno $(\mathrm{H})$ del carbón 5. (Cuadro № 1).

La Pregnan 3,20 Diona - se transforma en - Pregnan 3 Alfa, Ol 20
Ona - al cambiar en el carbón 3 su función cetona - O por la función alcohol - OH. Esta - Pregnan 3 Alfa Ol 20 Ona - puede tener su origen no sólo de la progesterona que acabamos de anotar, sino también de la desoxicorticosterona o cortexona.

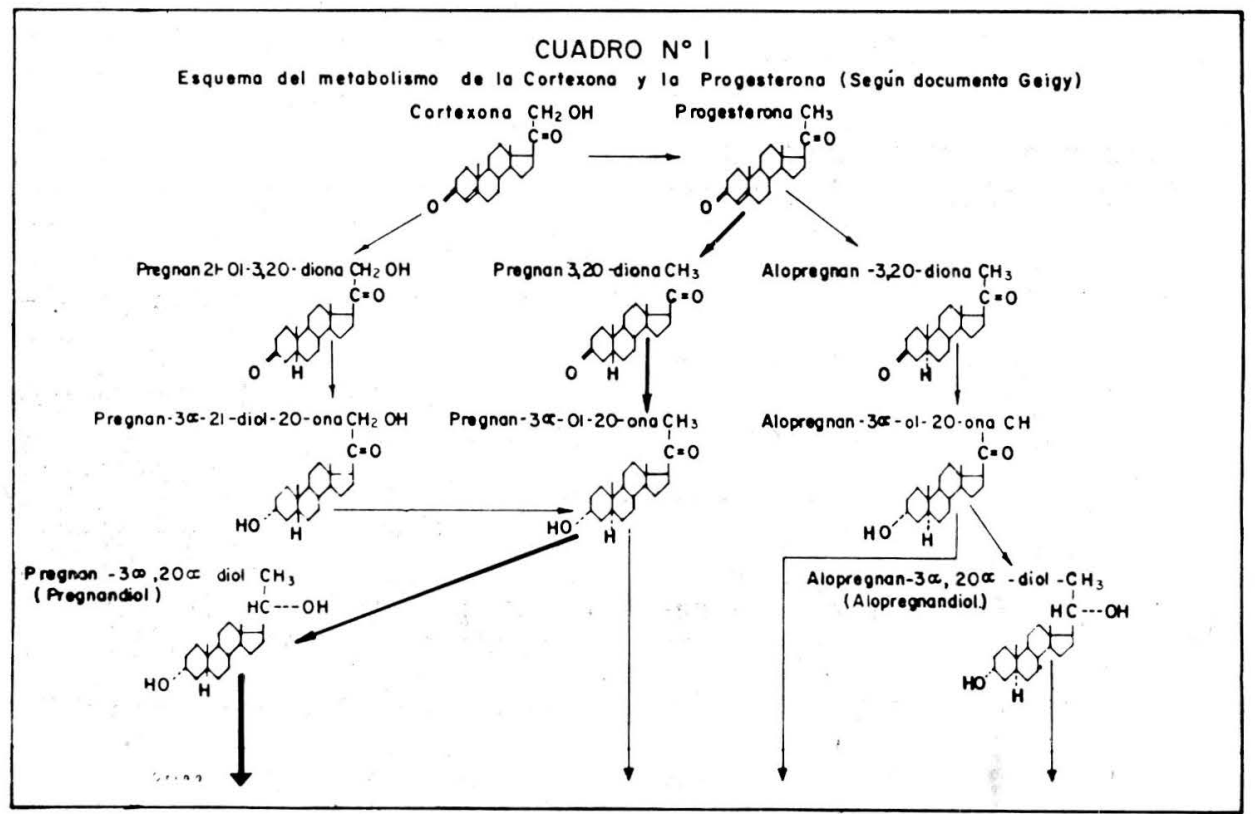

CUADRO No 1 - Esquema del metabolismo de la cortexona y de la progesterona hasta los productos finales que se encuentran en la orina. (Documento Geigy). (23)

El - Alopregnan 3, 20 Diona - se transforma en - Alopregnan 3 Alfa Ol 20 Ona - al cambiar en el carbón 3 su función cetona $=0$ por la función alcohol-OH. Tanto el - Pregnan 3 Alfa Ol 20 Ona - como el - Alopregnan 3 Alfa Ol 20 Ona - pueden encontrarse en dosis ínfimas en la orina. Sin embargo sus principales formas de eliminación se efectúan cambiando sus funciones cetónicas $=0$ del carbón 20 por una función alcohólica - $\mathrm{OH}$ - en este carbón, y así encontramos en la orina el producto final del metabolismo de la progesterona en los compuestos denominados Pregnan 3 Alfa 20 Alfa Diol - y - Alopregnan 3 Alfa 20 Alfa Diol - o más comunmente llamados - Pregnandiol y Alopregnandiol - y más genéricamente aún - Complejo Pregnandiol.

La proporción normal de Alopregnandiol a Pregnandiol es de 2/2 (1). En esquema ésta es la vía que sigue la progesterona en su metabolismo hasta llegar al pregnandiol, y ya hemos visto como éste puede tener su origen en la desoxicorticosterona y aún como lo demuestran los estudios de Arcos y colaboradores (2) con hidrógeno 3 y carbón 14 marcados puede proceder de la pregnenolona. Sinembargo para el médico que estudia la pregnandioluria de su paciente, la dosificación precisa de es- 
te esteroide tiene un gran significado clínico pues sus precursores cuando son diferentes a la progesterona no llegan a producir más de $0.01 \mathrm{mi}$ ligramos en la diuresis de 24 horas, y esta pequeña cifra no alcanza a modificar el concepto expresado por la dosificación hormonal.

b) Cantidad de eliminación: $\mathrm{Si}$ bien es cierto que los principales metabolitos de la progesterona se eliminan en forma de pregnandiol $y$ alopregnandiol, no podemos obtener en las dosificaciones urinarias más que unas cantidades muy pequeñas que oscilan según los autores y las técnicas de un $1 \%$ a un $30 \%$. Con la técnica cromatográfica de Waldi hicimos varias experiencias para de- terminar la cantidad de pregnandiol que obteníamos al aplicar diferentes dosis de progesterona en solución oleosa y en forma de capronato de 17 alfa-hidroxi-progesterona, en pacientes sin ciclos menstruales (con agenesias ováricas, o menopáusicas, u ovariectomizadas) y aun en pacientes masculinos. Los resultados fueron los siguientes:

En cuatro pacientes sin ciclos menstruales aplicamos una solución oleosa de 100 miligramos de progesterona por vía intramuscular y efectuamos dosificaciones del pregnandiol urinario diariamente hasta encontrar de nuevo las cifras de las dosificaciones previas (Cuadro № 2 ).

CUADRO № 2

Pregnandiol urinario obtenido de 100 Miligramos de Progesterona Parenteral En 4 pacientes sın ciclos menstruales. Promedio 8.9 Miligramos de Pregnandiol.

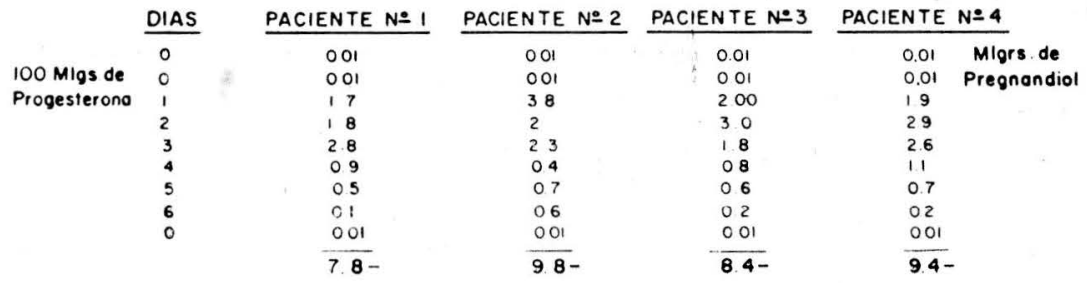

PROMEDIO 8.9 MLGRS

CUADRO Nọ 2 - Pregnandiol urinario obtenido en 4 pacientes sin ciclos menstruales, a las cuales se les aplicó 100 miligramos de Progesterona.

Como era lógico de esperar las eliminaciones no fueron constantes día a día pues en su absorción influye mucho las circunstancias locales de la inyección. La suma de los miligramos de pregnandiol nos dió como cifra máxima 9.8 y como mínima 7.8; el promedio fué de 8.9 miligramos.
Podemos decir que en general se obtienen 9 miligramos de pregnandiol, más menos 1 miligramo ( $\mathrm{sic}$ ) por cada 100 miligramos de progesterona que se inyecta.

Con el fin de comprobar una vez más esta constancia entre la proges- 
terona y el pregnandiol hicimos en 4 pacientes eumenorreicas dosificaciones en la fase progestacional y observamos que, la cifra máxima fué de 20.3 miligramos, y la mínima de 15.2 miligramos, para un promedio en las cuatro pacientes de 17.7 miligramos de pregnandiol; de acuerdo con los datos anteriores esta cifra corresponde a una producción aproximada de 200 miligramos de progesterona por ciclo, cifra que sabemos por pruebas de terapia sustitutiva en endometrio, es real. (Cuadro $\mathrm{N}$ ㅇ 3 ).

\section{CUADRO Ne 3}

\section{Pregnandicl urinario en la fase progestacional de 4 pacientes eumenorreicas. Promedio} total: 17.7 MLGRS. de Pregnandiol.

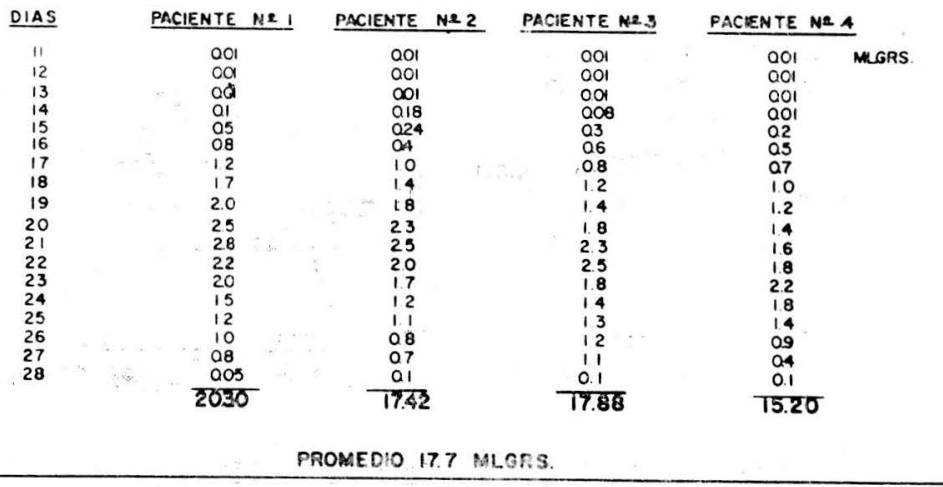

CUADRO No 3 - Cifras de pregnandiol urinario en 4 pacientes eumenorreicas. Dosificaciones diarias del día 11 al día 28 de los ciclos. Promedio $=17.7$ miligramos.

$Y$ para completar el estudio en lo referente a las cantidades de eliminación, hicimos dosificaciones en 4 hombres, aplicando en dos de ellos inyecciones intramusculares de progesterona en solución oleosa y en los otros dos aplicando inyecciones intramusculares de capronato de 17 alfa hidroxi-progesterona.

Los resultados nos muestran que en los hombres a los cuales les aplicamos la forma de capronato de 17 alfa hidroxi-progesterona en dosis de 250 miligramos, eliminaron en promedio 5.3 miligramos de pregnandiol más menos 0.1 miligramo. Es decir obtuvimos 2.1 miligramo de pregnandiol por cada 100 miligramos de progesterona. $Y$ en los hombres a los cuales les aplicamos 100 miligramos de progesterona pura obtuvimos en promedio 2.1 miligramos de pregnandiol. (Cuadro No 4).

Estas cifras difieren en forma clara y franca de la constante de $9 \mathrm{mi}$ ligramos de pregnandiol, por cada 100 miligramos de progesterona encontrada en las mujeres. En nuestro concepto este hecho se puede explicar si tomamos como base los trabajos de Davis y Plotz (3) con CO2 marcados, quienes demostraron que hay una fácil transformación de la progesterona en esteroides de 19 átomos de carbón y de una estructura muy probable androgénica, además Dorfman (4) explica en forma esquemática como es posible que la 


\section{CUADRO N24}

Pregnandiol urinario obtenido en 4 hombres a los cuales se les aplico capronato $17 \propto$ Hidroxiprogesterona y progesterona

\begin{tabular}{|c|c|c|c|c|c|c|}
\hline \multirow{2}{*}{$\begin{array}{l}100 \mathrm{Mlgrs} \text { de } \\
17 \propto \mathrm{Hidx} \\
\text { progesterono }\end{array}$} & DIAS & PACIENTE NN I & PACIENTE N 22 & PACIENTE N*3 & PACIENTE & $\begin{array}{l}\text { N2 } 4 \\
100 \text { Mlgrs de } \\
\text { Progesterona. }\end{array}$ \\
\hline & 0 & 001 & 001 & 001 & $\mathrm{OOI}_{-}$ & \\
\hline & 1 & 11 & 12 & 20 & 18 & \\
\hline & 2 & 09 & 10 & 02 & 02 & \\
\hline & 3 & 06 & 08 & 001 & 001 & \\
\hline & 4 & 05 & 0.4 & 001 & 0.01 & \\
\hline & 5 & 04 & 04 & $\overline{22}$ & 20 & TOTAL \\
\hline & 6 & 0.4 & $\begin{array}{l}04 \\
04\end{array}$ & & & \\
\hline & $\begin{array}{l}7 \\
8\end{array}$ & $\begin{array}{l}0.4 \\
0.4\end{array}$ & $\begin{array}{l}04 \\
04\end{array}$ & & & \\
\hline & 9 & 05 & 04 & & & \\
\hline & 10 & 001 & 001 & & & \\
\hline & TOTAL & 5. 2 & 54 & & & \\
\hline $\begin{array}{l}\text { PROA } \\
\text { POR }\end{array}$ & MEDIO: & $2.1 \mathrm{MLGRS}$ DE & $\begin{array}{l}\text { PREGNANDIOL } \\
\text { PROGESTERONA }\end{array}$ & $\begin{array}{l}\text { PROMEDIO }=2.1 \\
\text { POR CADA } 100\end{array}$ & $\begin{array}{l}\text { MLGRS. DE } \\
\text { MLGRS. DE }\end{array}$ & $\begin{array}{l}\text { PREGNANDIOL } \\
\text { PROGESTERONA }\end{array}$ \\
\hline POR & CADA K & WOMLGRS. DE & PROGESTERONA. & & & \\
\hline
\end{tabular}

CUADRO No 4 . Pregnandiol urinario en 4 hombres, a los cuales se les aplicó progesterona. Se obtuvo 2.1 miligramos de pregnandiol por cada 100 miligramos de progesterona.

progesterona se transforme en 17 alfa hidroxi-progesterona y luego en delta 4 androsten-3, 17 diona y luego en testosterona.

Pensamos nosotros que analizando las cifras de eliminación del pregnandiol en hombres a los cuales les hemos aplicado una cantidad determinada de progesterona, podremos valorar la actividad funcional de las células de Leydig, por la propiedad que estas células tienen de transformar la progesterona en compuestos androgénicos y así en los pacientes con una insuficiencia hormonal testicular se eliminarán cifras mayores de pregnandiol que las encontradas en los hombres normales, es decir la cantidad total de pregnandiol se inclinará más hacia las cifras femeninas de los 9 miligramos por cada 100 miligramos de progesterona, que hacia las cifras masculinas de los 2.1 miligramos de pregnandiol por cada 100 miligramos de progesterona, pues las células de Leydig de estas góna- das no podrán sino transformar parcialmente la progesterona en compuestos androgénicos y entonces una mayor cantidad de progesterona se metabolizará a pregnandiol.

Teniendo como base que un hombre adulto normal elimina, con esta prueba dinámica, 2.1 miligramos de pregnandiol cuando se le inyecta 100 miligramos de progesterona en solución oleosa, aplicamos en dos pacientes con hipofunción hormonal testicular 100 miligramos de progesterona, e hicimos dosificaciones diarias de pregnandiol urinario hasta encontrar las cifras de las dosificaciones previas a la inyección. (Estos dos pacientes son homosexuales y a ellos les habíamos efectuado un estudio de la actividad androgénica gonadal, por medio del fraccionamiento cromatográfico de los 17 cetoesteroides y ambos presentan una disminución marcada de la eliminación de androsterona y etiocolanolona, que revela la hipofunción hormonal testicular). 
Mayo-Junio 1967

Rev. Col. Obst. y Ginec.

La cantidad de pregnandiol dosificado fue de 11.2 en el primer paciente $y$ de 12.5 en el segundo, lo que nos muestra que las células de Ley-

dig de estos enfermos no habían transformado la progesterona en compuestos androgénicos. (Cuadro № 5 ).

\section{CUADRO Nㅡ5}

Pregnandiol urinario obtenido en 2 hombres homosexuales con hipofuncion hormonal testicular demostrada por fraccionamiento de 17 cetoesteroides. Se les oplicó 100 Miligra. mos de Progesterona.

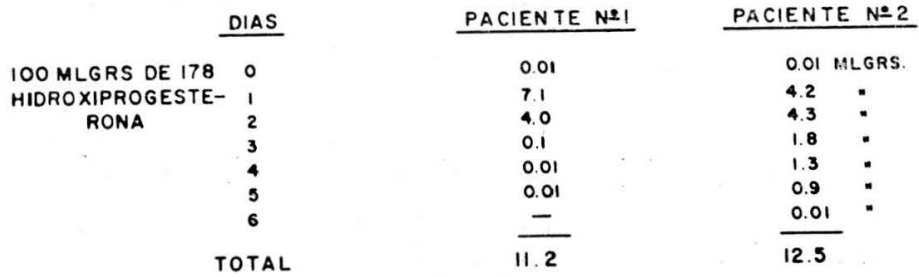

CUADRO No 5 - Pregnandiol urinario en 2 hombres con hipofunción hormonal testicular, a los cuales se les aplicó 100 miligramos de progesterona. Se obtuvo 11.2 y 12.5 miligramos de pregnandiol.

Para confirmar más esta prueba (Fig. № 1) que muestra una detendinámica, estudiamos un paciente ción de la espermatogénesis en el azoospérmico con biopsia testicular epitelio germinativo y en quien pre-

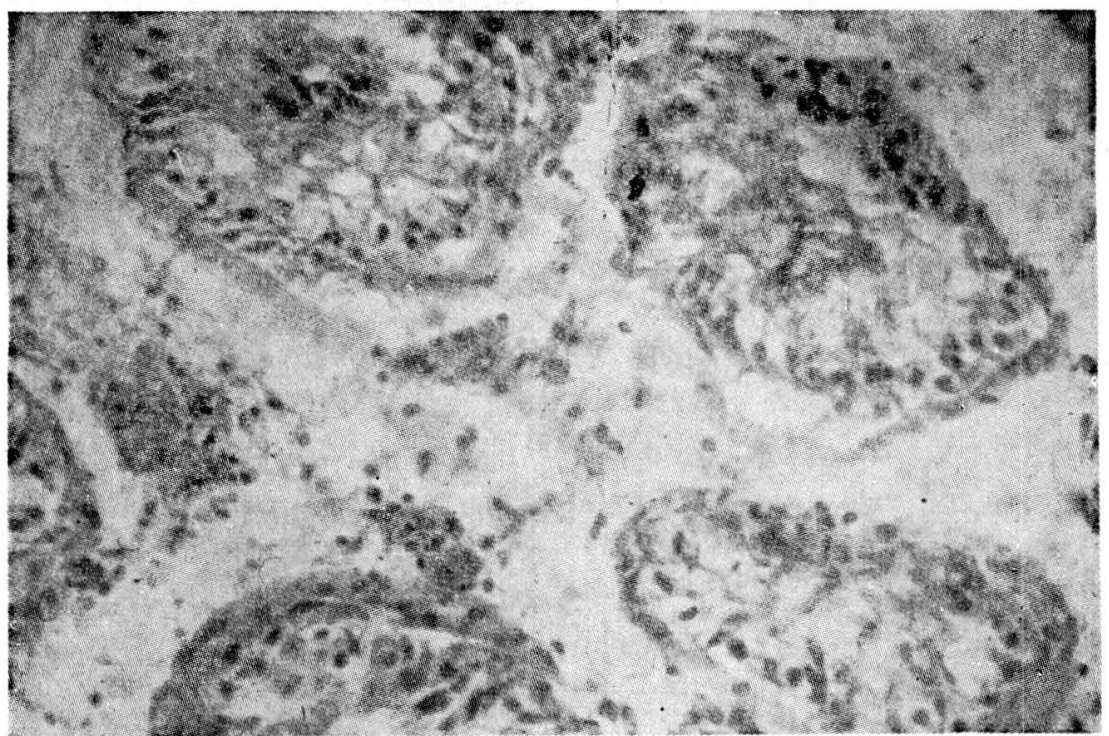

FIGURA No 1 - Biopsia testicular que muestra detención de la espermatogénesis en el epitelio germinativo. 
sumimos también una ligera hipofunción hormonal gonadal por una cifra baja de 17 cetoesteroides totales en la orina de 24 horas, de 10,5 miligramos.

En este paciente al aplicar $100 \mathrm{mi}-$ ligramos de progesterona en solución oleosa por vía intramuscular ob- tuvimos 3.4 miligramos de pregnandiol urinario, esta cifra se encuentra 1.3 miligramos por encima de los datos que hemos obtenido en los hombres normales estudiados, y concuerda con la presunción que teníamos de una ligera hipofunción hormonal testicular. (Cuadro № 6).

CUADRO № 6

Pregnandiol urinario en un hombre azoospérmico por detención de la espermatogénesis, con posible hipofucion hormonal Gonadal. Se le aplico 100 Miligramos de Progesterona.

\begin{tabular}{ll} 
DIAS & PACIENTE. \\
\cline { 2 - 2 } 0 & 0.01 \\
1 & 0.9 \\
2 & 11 \\
3 & 0.4 \\
4 & 0.9 \\
5 & 01 \\
6 & $\frac{0.01}{3.4}$ TOTAL
\end{tabular}

CUADRO No 6 - Pregnandiol urinario en un hombre azoospérmico por detención de la espermatogénesis, con posible hipofunción hormonal gonadal. Se le aplicó 100 miligramos de progesterona, se obtuvo 3.4 miligramos de pregnandiol.

La consideración de los casos anteriores nos inclina a pensar una vez más en la efectividad, de la dosificación del pregnandiol en hombres a los cuales se les aplicó progesterona, como una prueba dinámica para valorar la actividad hormonal del testículo. $Y$ creemos que esta prueba tiene también su aplicación en el campo de la endocrinología ginecológica, pues nos puede ayudar al estudio de la actividad hormonal del ovario, en lo referente a su producción de hormonas androgénicas, como en los hiperandrogenismos de las células del hilio y de las células tecales. c) Tiempo de eliminación: Para el estudio sobre el tiempo de eliminación de la progesterona en solución oleosa, hicimos en 4 pacientes dosificación del pregnandiol urinario después de aplicar 100 miligramos de progesterona. Encontramos que en promedio un $80 \%$ del pregnandiol se obtenía en la diuresis de los tres primeros días; además pudimos observar que las cifras de pregnandiol permanecieron seis días por encima de las dosificaciones previas a la inyección. Estos datos concuerdan con las experiencias de Plotz (5) quien usando progesterona con carbonos marcados encontró al 7: día cifras inferiores al $1 \%$ (Cuadro № 7). 
Promedio del tiempo y la cantidad de alimincción de 100 Miligramos de Progesterona aplicados

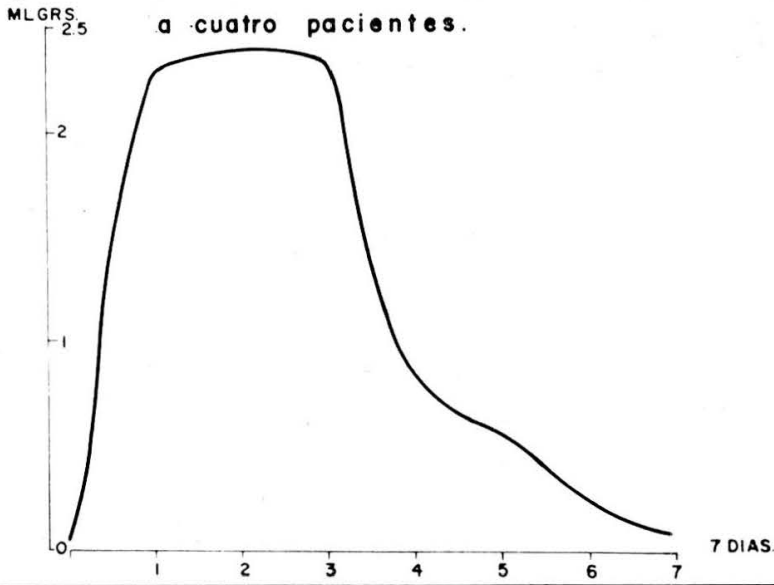

CUADRO No 7 - Promedio del tiempo y la cantidad de eliminación de 100 miligramos de progesterona aplicados a 4 pacientes.

Para investigar el tiempo de eliminación del capronato de 17 alfa hidroxiprogesterona, hicimos en cuatro pacientes la aplicación de 250 miligramos intramuscularmente y encontramos, que en promedio el máximo de eliminación se halló en el día 1 que su descenso se hacía en forma gradual y uniforme y que al $10^{\circ}$ día las cifras de eliminación son iguales a las iniciales. Estas experiencias revelan valores semejantes a los hallados por Plotz (5) quien al $10^{\circ}$ día encontró cifras inferiores al $1 \%$, usando progesterona con carbonos marcados. (Cuadro N: 8).

En nuestro concepto estas gráficas de eliminación son de gran ayuda para la clínica, pues nos muestran por métodos bioquímicos precisos el tiempo exacto de acción de las soluciones oleosas puras de progesterona y la de los capronatos de 17 alfa hidroxiprogesterona y no ya los métodos aproximados que se observan a través de los órganos efectores como son los cambios morfológicos de la colpocitología funcional y de la mucosa endometrial.

Existen estados patológicos en donde los conceptos generales sobre la biología de la progesterona y su metabolismo se encuentran alterados como en las hiperplasias suprarrenales; en estos casos las dosificaciones estáticas y las pruebas dinámicas inyectando progesterona son de gran ayuda para confirmar o esclarecer el diagnóstico. Lo anterior lo podemos ratificar con el siguiente caso que hemos estudiado:

C. S. de 29 años, soltera, con glándula mamaria de escaso desarrollo, hipertricosis y vello de distribución androide, con clítoris peniforme de unos 3 centímetros, no se aprecia cavidad vaginal, al tacto rectal útero pequeño y anexos normales con acentuada oligo o hipomenorrea a través del seno urogenital, cromatina sexual oral de $40 \% ; 17$ cetosteroides 31 miligramos en la diuresis de 24 horas, 17 hidroxiesteroides 5 miligramos, pregnandiol de 5 miligramos y pregnantiol de 1 miligramo. Se diagnosticó hiperplasia suprarrenal congénita y 


\section{CUADFO NQ 8}

\section{Promedio del tiempo y la contidad de eliminoción de 250 Miligramos de Capronato de 17 alfo Hidroxiprogesterona collicadas a cuetro pacientes.}

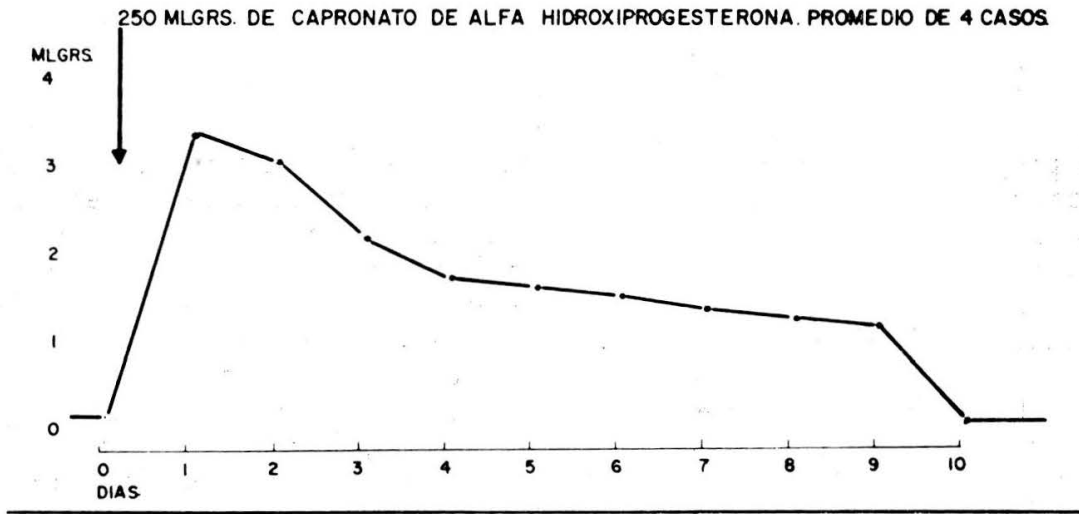

CUADRO No 8 - Promedio del tiempo y la cantidad de eliminación de 250 miligramos de capronato de 17 alfa hidroxiprogesterona aplicados a 4 pacientes.

se trató con prednisona y se corrigió quirúrgicamente la malformación externa; posteriormente se hizo eumenorreica, creció su glándula mamaria y contrajo matrimonio pudiendo tener relaciones sexuales normales, actualmente se halla en el quinto mes de gestación. Es obvio que el diagnóstico en este caso no se hizo por la sola dosificación del pregnandiol pero su resultado de 5 miligramos efectuado en medio de una amenorrea de 7 meses, fué una ayuda más en la confirmación de la hiperplasia suprarrenal.

\section{COMENTARIOS SOBRE LA TECNICA PARA LA DOSIFICACION DEL PREGNANDIOL DE WALDI}

Han sido muchas las técnicas que se han descrito para la dosificación del prenandiol desde que Marrian (6) en 1929 logró identificarlo en la orina de mujeres embarazadas. Entre los métodos utilizados el procedimiento de Vening y Browne (7) descrito en 1937 logra la dosificación de la sal sódica de glicuronato de pregnandiol por métodos gravimétricos, y posteriormente se le han hecho modificaciones a la técnica dosificando por colorimetría el ácido glucurónico del extracto.

Otro tipo de dosificación es el ideado en 1941 por Astwood y Jones (8) que logra por ebullición de la orina en medio ácido la extracción y purificación y luego la dosificación por método gravimétrico. Han sido logradas posteriores modificaciones en la extracción por medio de la betaglucuronidasa y reacción colorimétrica con el ácido sulfúrico. Estas técnicas tenían evidentemente utilidad clínica, pero su significado era más cualitativo que cuantitativo pues las cifras de recuperación oscilaban entre el $1 \%$ y el $30 \%$, y así los márgenes entre lo fisiológico y lo patológico eran tan difusos que las cifras obtenidas en una determinación carecían de valor para los autores como nos lo anota Williams (9), especialmente en las interpertaciones en el ciclo menstrual o en la etapa inicial del 
embarazo en donde son tan esenciales las dosificaciones que revelen fielmente los estados de hipoluteinismo.

El advenimiento de las técnicas cromatográficas ha permitido que hoy en día poseamos unos procedimientos tan exactos, que con razonable seguridad puedan darse las cifras de pregnandiol en la diuresis de 24 horas. Estos estudios se iniciaron con Klopper en 1955 (10) trabajando con cromatografías en columna y 8 nuevas técnicas se han creado con este procedimiento en estos 10 años.

En 1958 Eberlein (11) creó una nueva técnica cromatográfica en papel y en capa delgada de silicagela, y en 1962 Staub (12) y Waldi (13) crearon sus técnicas para dosificar pregnandiol basados en la cromatografía en capa delgada de Stall (14).
La técnica de Waldi (13) con la que elaboramos este trabajo en esencia consta de una ebullición de $20 \mathrm{cc}$. de orina obtenida de la diuresis de 24 horas a la cual se le agrega ácido clorhídrico, luego se extrae el pregnandiol con ciclohexano y se purifica con lavados con soda y agua, que se seca con sulfato de sodio anhidro; se evapora a baja temperatura preferiblemente al vacío y se disuelve el residuo en $0.5 \mathrm{ml}$. de cloroformo; de aquí se toma la muestra que se aplica en la placa de capa delgada de silicagela y se "elulle" en una solución de cloroformo-acetona, obteniendo así el fraccionamiento cromatográfico del pregnandial y las otras sustancias cromógenas; finalmente se desarrolla color con ácido o-fosfórico y ácido fosfomolíbdico frente a soluciones patrones conocidas. (Cuadro No. 9).

\section{CUADRO Nㅛ 9.}

Esquema para la dosificacion del Pregnandiol urinario Tec. de Woldi.

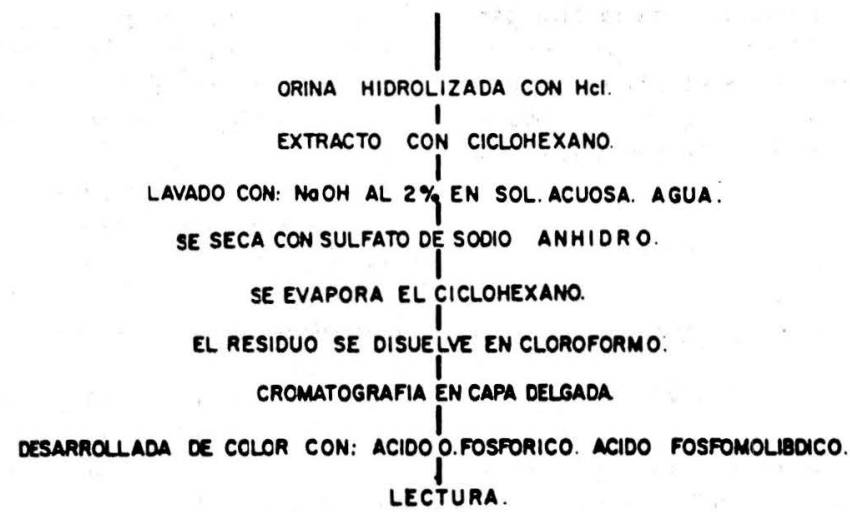

CUADRO No 9 - Esquema de la dosificación del pregnandiol urinario, según la téenica de Waldi.

Este procedimiento de Waldi cumple con los requisitos exigidos por la clínica al poderse demostrar en él, los cuatro factores que componen el "criterio de credibilidad" de una técnica, -ya expuestos por Dicsfaluzy 
(15). Analizaremos a continuación estos cuatro factores de precisión, exactitud, sensibilidad y especificidad, según nuestra propia experiencia.

a) Precisión: Se dice que una técnica es precisa cuando existe una variación mínima en dosificaciones replicadas. La prueba de ello la pode- mos obtener en análisis duplicados. Con la técnica de Waldi hicimos 5 dosificaciones duplicadas para investigar hasta donde este método de dosificación del pregnandiol urinario cumplía con el criterio de precisión. Pudimos observar que la diferencia máxima fué de 0.2 miligramos en la diuresis de 24 horas. (Cuadro № 10).

\section{CUADRO № 10}

\section{Resultados de 5 dosificaciones duplicadas de Pregnandiol urinario y las variaciones encontradas. Máxima variación 0.2 Miligramos.}

\begin{tabular}{|c|c|c|c|c|c|c|}
\hline MUESTRA & \multicolumn{2}{|c|}{ I- DOSIFICACION } & \multicolumn{2}{|c|}{22 DOSIFICACION. } & \multicolumn{2}{|c|}{ VARIACION } \\
\hline Ne I & 30 & MLGRS/24 H. & 311 & GRS $/ 24 \mathrm{H}$. & 0.11 & MLGRS. \\
\hline$N 2$ & 1.4 & " & 14 & $"$ & $\infty$ & * \\
\hline No 3 & 0.15 & $"$ & 0,0 & " & aos & $"$ \\
\hline Ne 4 & 0.2 & $"$ & 2.2 & " & 0.0 & $"$ \\
\hline Ne 5 & 1.7 & $\cdots$ & 1.9 & " & 0.2 & " \\
\hline
\end{tabular}

CUADRO No 10 - Dosificaciones duplicadas de pregnandiol urinario en 5 muestras y las variaciones obtenidas. Máxima variación 0.2 miligramos.

Por lo tanto podemos afirmar que para los fines de interpretación clínica esta técnica es precisa, ya que más o menos 0.2 miligramos es inferior a las variaciones que pudiésemos interpretar como hiper o hipoluteinismo en un caso dado.

b) Exactitud: Se dice que una técnica es exacta cuando existe consonancia de las medidas obtenidas, con los valores verdaderos. La prueba de ello la podemos obtener utilizando métodos de recuperación. Con la técnica de Waldi analizamos 6 muestras diferentes de orina $y$ en ellas hicimos de a dos dosificaciones, la primera para conocer la pregnandioluria de la muestra estudiada, y la segunda para conocer cuanto preghandiol se recuperaba en esta segunda muestra a la cual le habíamos agregado 100 gamas de pregnandiol. Observamos que la recuperación má- xima fué de 93 gamas y la mínima de 87 gamas. Analizando estos resultados vemos que el promedio de recuperación es del $90.5 \%$ (Cuadro № 11 ).

Como dato curioso comenta Waldi en su técnica que cuando se hacen pruebas de recuperación utilizando agua destilada para la hidrólisis ácida en vez de orina, se destruye en la elaboración del proceso de un $65 \%$ a un $70 \%$ del pregnandiol agregado a la muestra, y por razones aún no estudiadas la orina protege al pregnandiol de esta destrucción, y así trabajando con orina en vez de agua destilada es posible recuperar un $90 \%$ de la cantidad agregada. Nosotros hicimos esta experiencia en 4 oportunidades y con agua destilada solo pudimos recuperar un $40 \%$ del pregnandiol que habíamos adicionado a la muestra. 
CUADRO N2II

Valores de recuperaciones en 6 dosificaciones de Pregnandiol urinario; promedio de recuperación $90.5 \%$

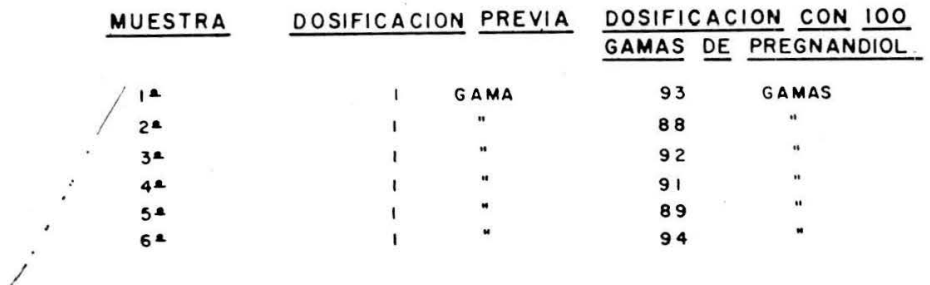

PROMEDIO DE RECUPERACION $90.5 \%$

CUADRO No 11 - Dosificaciones de pregnandiol urinario, en 6 muestras diferentes, antes y después de agregar 100 gamas de pregnandiol a cada una de ellas. Promedio de recuperación $90.5 \%$.

c) Sensibilidad: Se dice que una técnica es sensible cuando es mínima la cantidad detectable que difiere significativamente de cero. La prueba de ello lo podemos obtener con experiencias de recuperación en muestras con niveles bajos. Como base para estudiar la sensibilidad encontramos que un grupo de orinas estudiadas tenía siempre una cantidad aproximada de 10 gamas en la diuresis de 24 horas. Para investigar si era posible dosificar cantidades me- nores de estas 10 gamas, hicimos con la técnica de Waldi en 4 casos diluciones de esas orinas y pudimos constatar, que cantidades menores de 10 gamas no pudieron ser detectadas. De donde podemos afirmar que la mínima cantidad detectable en la diuresis de 24 horas es de 10 gamas y por lo tanto para fines de interpretación clínica esta técnica cumple ampliamente los requerimientos de sensibilidad. (Cuadro № 12).

\section{CUADRO NR 12}

\section{Dosificación de Pregnandiol urinario en orinos con 10 gamas en la Diuresis de 24 horas, $y$ en diluciones acuosas de éstas orinas}

MUESTRA

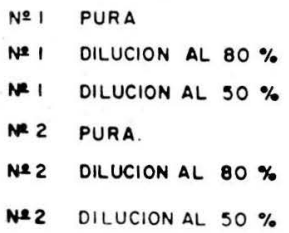

DOSIFICACION

O OI MILIGRAMO

MENOS DE O.OI MLGRS.PERO NO DOSIFICABLE NEGATIVO

OO MILIGRAMOS.

POSITNO PERO NO DOSIFICAELE.

NEGATIVO

CUADRO No 12 - Dosificación de Pregnandiol urinario en orinas con 0.01 miligramos en diuresis de 24 horas, y los resultados de las diluciones acuosas de estas orinas. 
d) Especificidad: Se dice que una técnica es específica cuando las sumas de las evidencias físicas, químicas, y biológicas indican identidad. La prueba de ello lo podemos obtener con estimación de algunas constantes físicas y características biológicas y químicas. Con la técnica de Waldi hicimos varias dosificaciones con esteroides patrones conocidos como el pregnandiol, el pregnantriol, el alo-pregnantriol, la estrona, el estriol, el estradiol, y la androsterona. Pudimos comprobar que estos esteroides tienen un "RF" constante en la "elución" con acetona-cloroformo, en la proporción 1 a 9 y que al "correr" la mezcla en la placa de capa delgada de silicagela hasta una altura de 10 centímetros y desarrollar color con ácido o-fosfórico y ácido fosfomolíbdico, el pregnandiol se encuentra a 4.1 centímetros de su punto inicial, el pregnantriol a 3.1 centímetros, el alo-pregnandiol a 6.0 centímetros. La estrona, a 10.0 el estriol, a 1.3 y el estradiol a 9.0 centímetros, y la androsterona a 8.5 centímetros. Por lo tanto la evidencia física del "RF" constante de la "elución", sumada a la química del color obtenida en presencia de los ácidos mencionados, nos permiten afirmar que esta técnica es específica, y por consiguiente tendrán valor clínico sus dosificaciones. (Fig. № 2).

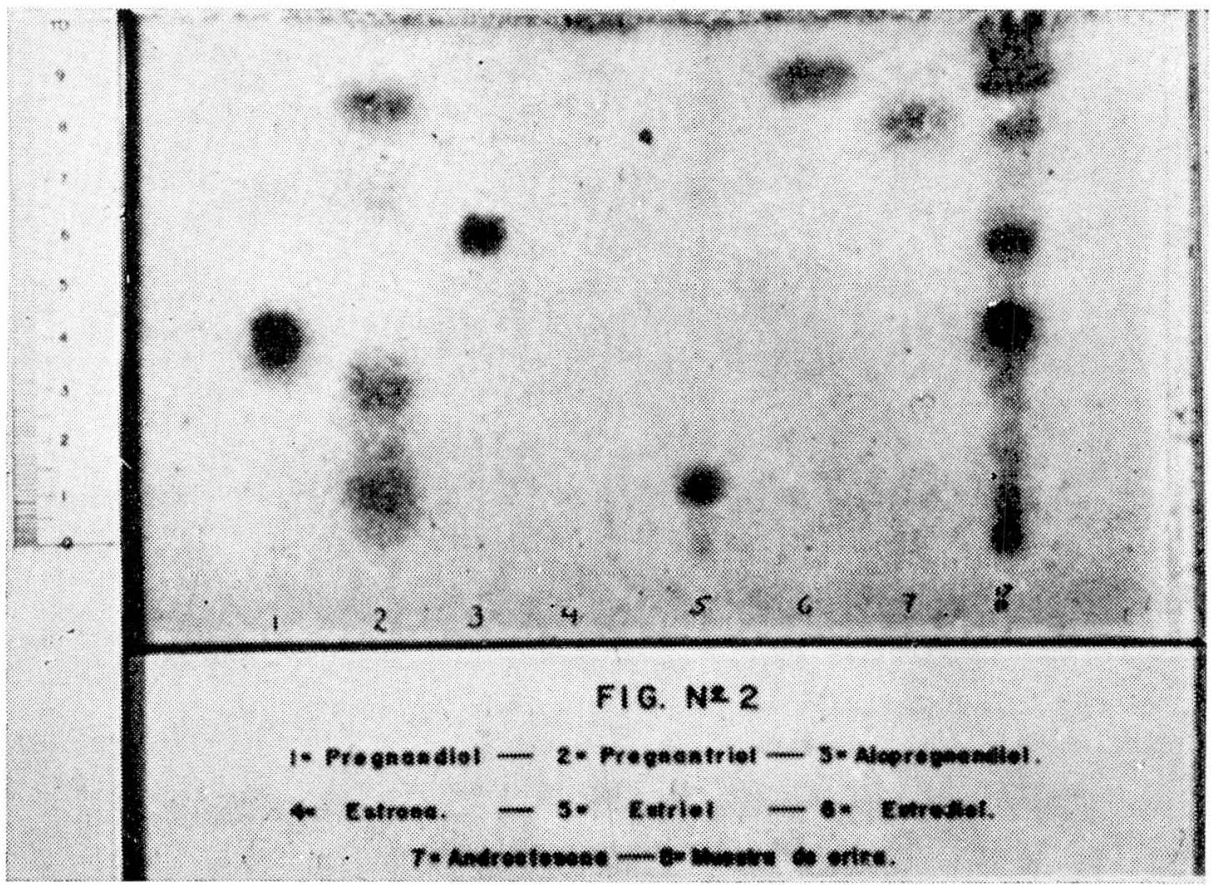

FIGURA No 2 - Color desarrollado con ácido ó-fosfórico y ácido fosfomolíbdico en placa de silicagela después de "elución" en acetona cloroformo ( 1 a 9) hasta 10 centímetros por los siguientes esteroides: $1=$ pregnandiol, $2=$ pregnantriol, $3=$ alo-pregnandiol, $4=$ estrona, $5=$ estriol, $\mathbf{6}=$ estradiol, $7=$ androsterona, y $8=$ muestra de orina. 
La técnica de Waldi posee una serie de condiciones favorables que enumeraremos a continuación y que en nuestro concepto la hacen ideal para nuestro medio. Son ellas:

19) Cumple con los requisitos analizados del "criterio de credibilidad", que son la base fundamental de toda buena técnica y que hacen posible la aplicación en la clínica de las cifras encontradas en la diuresis, y más concretamente en los valores subnormales.

2) Es una técnica rápida que puede efectuarse en 3 horas, lo que hace posible que en determinados casos se puedan hacer controles diarios de la pregnandioluria.

3) Después de montada la técnica es posible que las dosificaciones sean efectuadas por técnicas de laboratorio, claro está con supervigilancia y control de un médico, o de un bioquímico.

49) Los reactivos de una dosificación no tienen un costo superior a los \$20,00.

5․) El material empleado puede usarse numerosas veces y su desgaste es mínimo, su costo inicial es de unos $\$ 5.000,00$.

69) Las cifras obtenidas son de fácil interpretación clínica, y

7․) Los guarismos de la pregnandioluria permanecen reales cuando la paciente toma progestógenos del tipo nor-esteroides, pues estos últimos no se eliminan en forma de pregnandiol (24).

\section{ANALISIS DE LAS CIFRAS DE PREGNANDIOL URINARIO EN EL CICLO MENSTRUAL, EL EMBARAZO $Y$ LA AMENAZA DE ABORTO}

\section{Ciclo Menstrual}

Las técnicas antiguas para dosificar el pregnandiol urinario, los estudios bioquímicos y morfológicos del endometrio y la citología exfoliativa vaginal, nos indicaban que el pregnandiol iniciaba su ascenso de eliminación a partir del día 14 del ciclo y que este curso declinaba uno o dos días antes de la menstruación.

Cuando Klopper (16) montó su técnica cromatográfica para la dosificación del pregnandiol en 1957, mostró que el máximo de eliminación se encontraba hacia la mitad de la fase luteínica y que luego las cifras descendian en forma paulatina.

Nosotros hicimos estudios en 4 pacientes eumenorreicas y encontramos, al sacar una gráfica con el promedio de las 4 pregnandiolurias, con esta técnica de Waldi que el máximo de eliminación se encontraba en el día 21 del ciclo. Nuestra gráficas difieren de las de Klopper (16) en cuanto a los miligramos diarios y así en la fase estrogénica nuestras cifras son de 0.01 miligramos en cambio el promedio de Klopper es de 1.2 miligramos. En la fase progestacional nuestras cifras promedio no pasan de 2,3 miligramos, en cambio para Klopper las cifras máximas llegan a más de los 4 miligramos diarios. En nuestro concepto estas diferencias en la cantidad de eliminación se deben a una mayor depuración técnica. (Cuadro № 13).

Tomando como base estas cifras de la fase progestacional hicimos en 6 pacientes con historia clínica que nos indicaba insuficiencia luteínica, dosificaciones seriadas y encontramos que en tres de ellas no se aprecia curva ascendente lo que nos revela ciclos anovulatorios y en las otras 


\section{CUADRO NN 13}

Pregnandiol urinario en el ciclo menstrual.

Gráfica de Klopper- un caso- (En línea)

Grófica nuestra Promedio de 4 casos(En puntos).

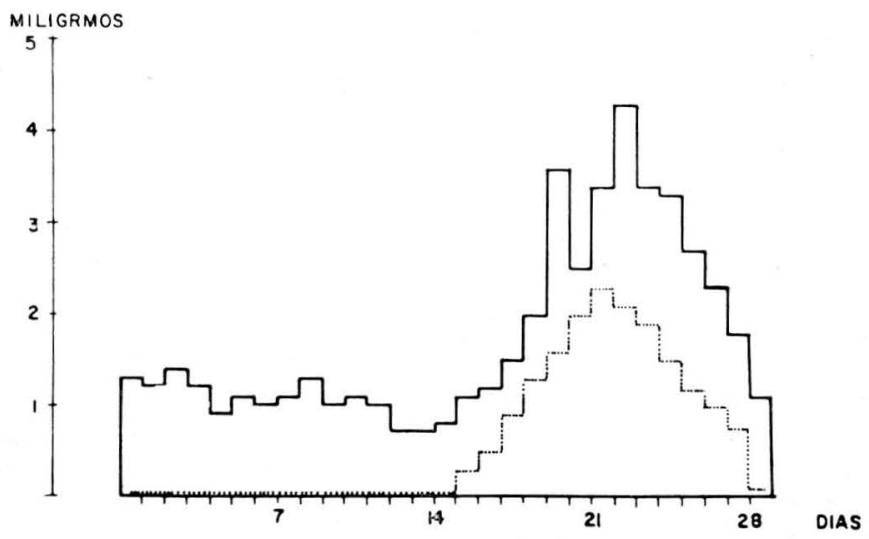

tres pacientes las cifras de elimina- curvas que revelan ovulación pero ción de pregnandiol nos muestran cuya cantidad total de pregnandiol se claramente los hallazgos típicos de la encuentra muy inferior a la media insuficiencia luteínica parcial con normal nuestra. (Cuadro № 14).

\section{CUADRO N2 14}

Pregnandiol urinario en 3 pacientes con insuficiencia luteinica.

Curva de la media normal nuestra. (Línea gruesa)

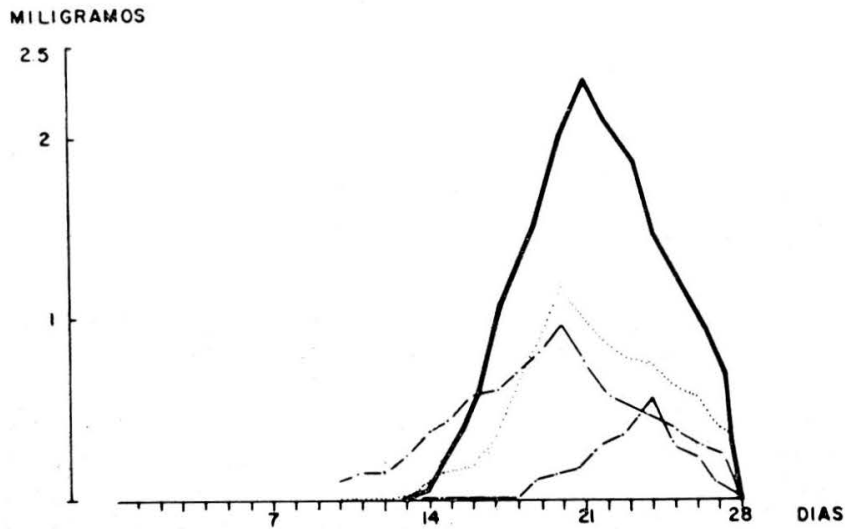


Consideramos que en el estudio del ciclo menstrual, estas dosificaciones tienen una gran aplicación clínica, pues no solo nos revelan la existencia de estados de hipofunción luteínica sino que nos orienta con las dosis terapéuticas en casos de terapia sustitutiva, en especial en problemas de desequilibrio estrógeno-progesterona como en las mastosis y en los trastornos de nidación.

\section{Embarazo}

Muchas han sido las curvas que se han publicado de la eliminación de pregnandiol en el embarazo y todas ellas expresan cifras que se encuentran muy por encima de las obtenidas con las nuevas y depuradas técnicas. Ramírez Soto, Castelazo Ayala y col. (17), presentaron en 1964 una curva de pregnandiol en el embarazo utilizando la técnica cromatográfica de Goldzieher y Nakameva (18) en donde las cifras aparentemente muy bajas son en todo semejantes a las que hemos obtenido con la técnica de Waldi.

Queremos hacer énfasis en las cifras del principio del embarazo en las cuales ni en los 11 casos estudiados por Ramírez y Castelazo (17) ni en los 10 casos estudiados por nosotros, se obtuvieron cantidades mayores de los 5 miligramos de pregnandiol en las primeras 6 semanas de la gestación. Hemos tenido oportunidad de efectuar en dos pacientes dosificaciones 48 horas antes del parto y hemos encontrado que su eliminación es muy baja, de 3.2 y 4 miligramos en la diuresis de 24 horas. (Cuadro № 15).

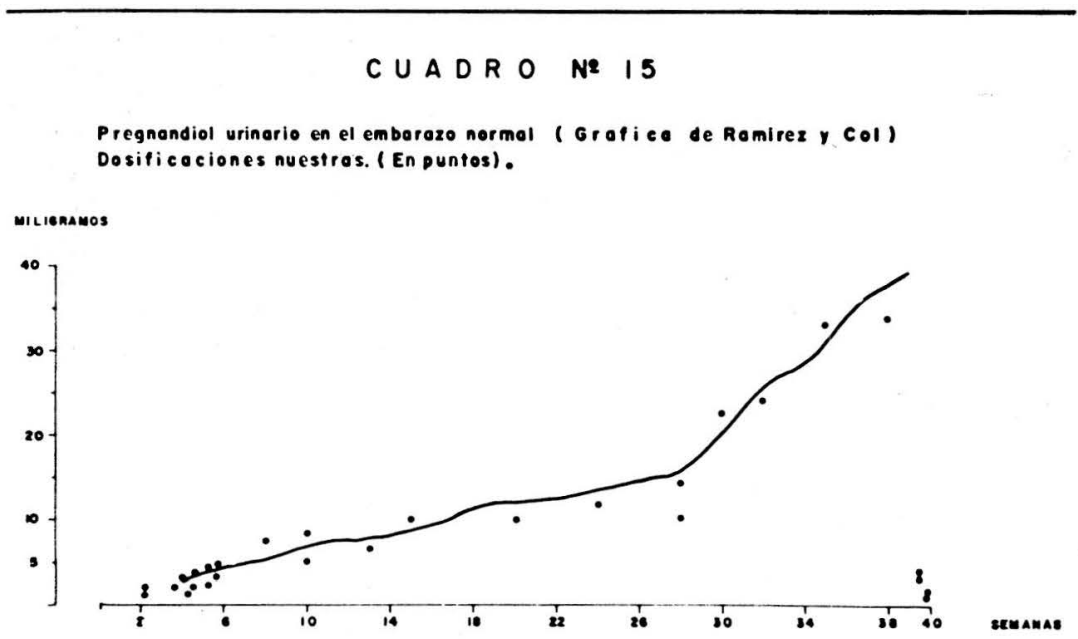

CUADRO No 15 . Pregnandiol urinario en el embarazo normal. Gráfica de Ramírez y col. Dosificaciones nuestras. (En puntos).

En otros dos casos hicimos dosificaciones 24 horas antes del parto $y$ encontramos cifras de 1.2 y 1.5 miligramos en la diuresis de 24 horas.
Prácticamente las dosificaciones que hicimos en las diferentes semanas del embarazo nos mostraron concordancia con la curva obtenida por 
Ramírez Col. cifras de embarazos normales. Consideramos que la mayor utilidad de la dosificación de la pregnandioluria en las diferentes semanas de la gestación, la tenemos en la patología del embarazo relacionada con el aborto de origen hormonal, en estos casos la dosificación nos revela la presencia de una insuficiencia en la producción de progesterona y nos orienta en la dosis diaria requerida de dicha hormona en la terapia sustitutiva; además nuevos campos de investigación se vislumbran con el estudio de la pregnandioluria en el terreno de la toxemia gravídica como lo anota Watteville (19).

\section{Amenaza de Aborto}

Basados en los trabajos de Karnaky (20) sobre la acción del estilbestrol en el aborto habitual y en los estudios de los Smith (21) sobre la acción de los estrógenos oxidados sobre el trofoblasto, y en la prueba citológica de Watteville (22) de la acción hormonal progestacional del benzoato de estradiol en las amenazas de aborto, hicimos un estudio de 13 casos con cuadro clínico de amenazas de aborto tratados con etinil estradiol y los resultados fueron los siguientes:

a) Un grupo de 5 pacientes con cuadro clínico de franca amenaza de aborto presentaron un pregnandiol urinario inicial que no llegó a los 2.0 miligramos en las 24 horas. En unos pocos casos esta cifra subió menos de 0.5 miligramos en los primeros días, pero luego a pesar de la terapia con dosis de 0.4 miligramos de etinil estradiol diarios, estas cifras bajaron hasta 0.1 miligramos de pregnandiol y terminaron en aborto. Uno de los casos clínicamente evolucionó en forma satisfactoria sin hemorragia ni contracciones, pero el pregnandiol persistió en $0.01 \mathrm{mili}$ gramos diarios y su final fué un aborto frustro confirmado a los 4 meses de iniciada la gestación.

b) Un grupo de tres pacientes con sintomatología de amenaza de aborto pero con cifras iniciales de pregnandiol superiores a los 2.5 miligramos en la diuresis de 24 horas, fué tratado con dosis no superiores a los 0.2 miligramos de etinil estradiol por día. En estos casos observamos que las cifras de pregnandiol subían con respecto a la dosificación inicial pero no llegaron estas pacientes en un tiempo prudencial de tres semanas a las cifras medias normales del embarazo. Una terapia posterior y más intensa fué necesaria para alcanzar las cifras normales del pregnandiol indicadas en la curva de Ramírez y col. (17).

c) Un grupo de 5 pacientes con sintomatología de amenaza de aborto $\mathrm{v}$ con cifras iniciales de pregnandiol superiores a los 2.5 miligramos diarios, fué tratado con dosis de 0.4 miligramos de etinil estradiol por día y encontramos que en 4 casos las cifras llegaron a los niveles de la media normal y que la sintomatología de amenaza desapareció. Controles posteriores indican que estos embarazos bajo punto de vista clínico y de la eliminación del pregnandiol urinario continúan su curso normal.

El conjunto de enfermas estudiadas con amenaza de aborto de origen hormonal, nos induce a las siguientes consideraciones:

19) Las amenazas de aborto con cifras de pregnandiol por debajo de los 2.0 miligramos en la diuresis de 24 horas, son de mal pronóstico.

2:) Las dosis de 0.2 miligramos de etinil estradiol por día son insuficientes para el tratamiento de la 
amenaza de aborto de origen hormonal.

3) La terapia estrogénica con dosis de 0.4 miligramos por día de etinil estradiol es efectiva en las amenazas de origen hormonal.

4․) La terapia estrogénica en las amenazas de aborto de origen hormonal aumenta la producción de progesterona endógena, como lo prueban los controles del pregnandiol urinario.

\section{RESUMEN}

Se hacen algunas consideraciones biológicas sobre la progesterona y el pregnandiol y se presenta una prueba dinámica para valorar la actividad androgénica de las gónadas. Se analiza la técnica cromatográfica de Waldi para la dosificación del pregnandiol urinario bajo el punto de vista del "Criterio de credibilidad". Se presentan las curvas normales del pregnandiol urinario en el ciclo menstrual y el embarazo y los hallazgos en la amenaza de aborto de tipo hormonal, con terapia estrogénica.

\section{BIBLIOGRAFIA}

1 BILBAO A., FILGUEIRA F., ARDANAZ J., PINTO B.: Ausencia de pregnandiol en la orina de los pacientes con insuficiencia hepática grave. Rev. Clin. Esp. 93: 366372, 1964.

2 ARCOS M., GURDIPE E., WIELE R. L. V., LIEBERMAN S.: Precursors of urinary pregnandiol and their influence on the determination of the secretory rate of progesterone. J. Clin. Endocrinol. Metab. 24: $237-$ 245, 1964.

3 DAVIS M. E., PLOTZ J.: The Metabolism of progesterone and its clinical use in pregnancy. Recent. Progr. in Hormone Res. N. Y. 13: 347-379, 1957.

4 DORFMAN R. I.: Metabolism of androgens, estrogens and corticoids. Amer. J. Med. 21,5: 679-687, 1956.
5 PLOTZ E. J.: Deusche Endokrinologen. Tagung, Kiel. 1959.

6 MARRIAN G. F.: Chemistry of oestrin ; preparation from urine and separation from unidentified solid alcohol. Biochem J. 23 : 1090-1098, 1929.

7 VENNING E. H., BROWNE J. B. L.: Studies on corpus luteum function; urinary excretion of sodium pregnandiol glucuromidate in human menstrual cycle. Endocrinology, 21: 711-821, 1937.

8 ASTWOOD E. B., JONES G. F. S.: Simple method for quantitative determination of pregnandiol in human urine. J. Biol. Chem. 137: 397-407, 1941.

9 WILLIAMS R. H. Tratado de Endocrinología. 887. Salvat Ed. Barcelona 1959.

10 KLOPPER A. I., MICHIE E. A., BROWN J. B.: A method for the determination of urinary pregnanediol. J. Endocr. Lond. 12 (3) : 209-219, 1955.

11 EBERLEIN W. R., BONGIOVANNI A. M.: A paper chromatographic method for the measurement of pregnanediol in urine. J. Clin. Endocr. Metab. 18 (3): 300-308, 1958.

12 STAUB M. C., GAITAN E., DINGMAN J. F.: A simple method for the determination of urinary pregnanediol and pregnanetriol by glass fiber paper chromatography. J. Clin. Endocr. 22: 87-92, 1962.

13 Einfache quantitative Schnellbestimmung von Pregnandiol auf Dünnschichtplatten zum Frühschwangerschaftsnachweis und zur Uberwachung des Cyclus bei der Frau. Klin. Wschr. 40: 827-830, 1962.

14 STAHL E.: Thin-layer chromatography 11. Standarization, detection, documentation and application. Chemiker. Z.tg. 82: 323329, 1958.

15 DICSFALUZY E.: Chemical determination of oestrogens in the urine. Acta Endocr. Kbh. 24 (Suppl. 31): 11-26, 1957.

16 KLOPPER A. I.: The excretion of pregnanediol during the normal menstrual cycle. J. Obst. Gyn. Brit. Empire 64: 504-511, 1957.

17 RAMIREZ SOTO E., CASTELAZO AYALA L., CORRAL GALLARDO J., ALTAMIRANO M.: Memorias $V$ Reunión anual Soc. Mex. Nutr. Endocr. 31, 1964. 
18 GOLDZIEHER J. W., NAKAMEUA G.: A clinical method for the determination of urinary pregnanediol and pregnanetriol. Acta Endocr. (Koben havn) 41: 371-380, 1962.

19 WATTEVILLE H.: Pregnandiol determinations in the clinic and in research. J. Clin. Endocr. 11: 251-266, 1951.

20 KARNAKY J. K.: Use of stilbestrol for treatment of threatened and habitual abortion and premature labor; preliminary report. South M. J. 35: 838-847, 1942.

21 SMITH O. W., SMITH G. V. S., HURWITZ D.: Increased excretion of pregnanediol in pregnancy from diethylstilbestrol with spe- cial reference to prevention of late pregnancy accidents. Am. J. Obst. Gynec. 51: $411-415,1946$

22 WATTEVILLE $H_{\text {.: }}$ Valoración de las pruebas de laboratorio en la conducta clínico terapéutica del aborto. Memorias del III Congreso Mexicano de Obst. y Gynec. 35, 1958

23 Documenta Geigy Tablas científicas, 195. Impreso por la Sociedad Alianza de Artes Gráficas (S.A.D.A.G.). Barcelona. 1958.

(Traducido de la reimpresión, revisada, de la quinta edición alemana).

24 LITTER M.: Farmacología. 968. Ed. "El Ateneo". Buenos Aires, 1964. 